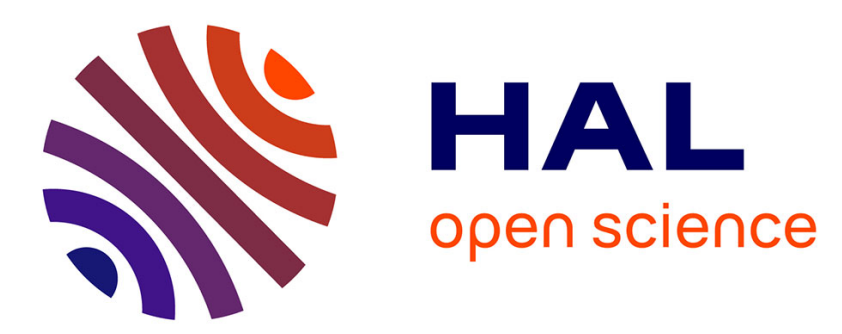

\title{
Enhanced active motion of Janus colloids at the water surface
}

Xiaolu Wang, Martin In, Christophe Blanc, Maurizio Nobili, Antonio Stocco

\section{To cite this version:}

Xiaolu Wang, Martin In, Christophe Blanc, Maurizio Nobili, Antonio Stocco. Enhanced active motion of Janus colloids at the water surface. Soft Matter, 2015, 11 (37), pp.7376-7384. 10.1039/c5sm01111f . hal-01216307

\section{HAL Id: hal-01216307 https://hal.science/hal-01216307}

Submitted on 11 Mar 2019

HAL is a multi-disciplinary open access archive for the deposit and dissemination of scientific research documents, whether they are published or not. The documents may come from teaching and research institutions in France or abroad, or from public or private research centers.
L'archive ouverte pluridisciplinaire HAL, est destinée au dépôt et à la diffusion de documents scientifiques de niveau recherche, publiés ou non, émanant des établissements d'enseignement et de recherche français ou étrangers, des laboratoires publics ou privés. 


\title{
Enhanced active motion of Janus Colloids at the water surface
}

Xiaolu Wang, ${ }^{1}$ Martin In, ${ }^{1}$ Christophe Blanc, ${ }^{1}$ Maurizio Nobili, ${ }^{1}$ Antonio Stocco ${ }^{1,2} *$

${ }^{1}$ Laboratoire Charles Coulomb (L2C), UMR 5221 CNRS-University of Montpellier, Montpellier F-34095, France.

${ }^{2}$ DWI - Leibniz-Institut für Interaktive Materialien, Aachen 52056, Germany. E-mail: Antonio.Stocco@umontpellier.fr

Keywords: self-propulsion, interface, Janus particles, diffusions, partial wetting.

\begin{abstract}
We have investigated the active motion of self-propelled colloids confined at the air-water interface and explore the possibility of enhancing the directional motion of self-propelled Janus colloids by slowing down their rotational diffusion. The two dimensional motion of micron-sized Silica-Platinum Janus colloids has been experimentally measured by particle tracking video-microscopy under increasing concentration of the catalytic fuel, i.e. $\mathrm{H}_{2} \mathrm{O}_{2}$. Compared to the motion in bulk, a dramatic enhancement of both the persistence length of trajectories and the speed has been observed. The interplay of colloid self-propulsion, due to an asymmetric catalytic reaction occurring on the colloid, surface properties and interfacial frictions controls the enhancement of the directional movement. The slowing down of the rotational diffusion at the interface, also measured experimentally, plays a pivotal role in the control and enhancement of active motion.
\end{abstract}

\section{Introduction}

Active colloids are an emerging and promising class of colloidal particles which are designed to perform autonomous motion by transforming chemical or other form of energies into work and propulsion. ${ }^{1,2,3,4}$ New lab-made colloids can be now designed not only tailoring precisely the size, surface functionalities and interactions but also rendering their motion 
directional and controllable, overcoming the limitation of random Brownian walks. ${ }^{5,6,7}$ In this regard, those active colloids are currently investigated as promising systems able to perform specific tasks such as transport $\operatorname{cargos}^{8,9}$ and degrading water contaminants. ${ }^{10,11}$

Several strategies have been envisioned to control the motion of active colloids. External magnetic ${ }^{12}$ and electric fields ${ }^{13}$ have been exploited to control the motion of different Janus particles. Field gradients have been also investigated theoretically to control directional motion of active colloids emulating the bacteria movement, which is directed by nutrient concentration gradient. ${ }^{14}$ Pinchasik et al. recently showed how active colloids can use biomimetic principles to move close to a water interface and perform two or three dimensional movement depending on the nature of the interfacial forces. ${ }^{15}$

In all the above mentioned cases, it is worth noting that directional active motion is coupled with the Brownian rotational diffusion. Similar to run-and-tumble systems, active colloids show a diffusive behavior at large length and time scales, and an effective diffusion coefficient $D_{\text {eff }}$ can be defined as: ${ }^{16,17}$

$D_{e f f}=D_{T}+V^{2} /\left(4 D_{R}\right)$,

where $D_{T}$ and $D_{R}$ are respectively the Brownian translational and rotational diffusion coefficients and $V$ is the self-propulsion velocity. Both the passive component $D_{T}$ and the active component $V^{2} /\left(4 D_{R}\right)$ of the motion contribute to an overall effective diffusion. Thus, only at relatively high speed $V$ (of the order of some $\mu \mathrm{m} / \mathrm{s}$ for micron sized particles in aqueous solution) or at short time an active motion can be distinguished from passive Brownian diffusion.

Note that in the run-and-tumble model, translational Brownian diffusion coefficient $D_{T}$ is neglected and $D_{e f f}=V^{2} \tau / d$, where $\tau$ is the tumbling time and $d$ is the dimensionality. ${ }^{16}$ Accounting for the latter difference, the run-and-tumble and active colloid dynamics are equivalent; and the tumbling time corresponds to the rotational diffusion time $\tau_{\mathrm{R}} \approx 1 / D_{R}{ }^{16}$ 
As far as directional and active transport is concerned, a major challenge consists in obtaining the longest possible directional trajectories while minimizing random Brownian motion. This target can be achieved either by attaining high $V$ or by reducing $D_{R}$ (see Equation 1).

The slowing down of the rotational diffusion is clearly of primary importance for enhancing directional movements. This is particularly true for active systems whose sizes are in the micron and submicron ranges since $D_{R}$ scales with the inverse of the cube of the size. ${ }^{18}$ Such slowing down could be efficiently achieved by confining the rotational, while not hindering the directional self-propulsion.

A well-studied example of self-propelled particles is Janus particles half covered by platinum where the active motion is due to catalytic reaction occurring on the platinum region of the colloid, which transforms $\mathrm{H}_{2} \mathrm{O}_{2}$ in water and oxygen. ${ }^{17}$ The velocity $V$ depends on the $\mathrm{H}_{2} \mathrm{O}_{2}$ concentration (the fuel of the catalytic engine), the size of the colloid ${ }^{19}$, the thickness of the platinum layer ${ }^{20}$ and on the reaction and transport phenomena occurring on the particle's surface. ${ }^{21}$ In bulk, for particles of about $2 \mu \mathrm{m}$ diameter, $V \approx 9 \mu \mathrm{m} / \mathrm{s}$ have been measured for polystyrene-Pt colloids ${ }^{19}$ and for silica-Pt colloid $V$ could be as high as $6 \mu \mathrm{m} / \mathrm{s}$ when the catalytic fuel concentration is $5 \% .^{22}$

In this contribution, we investigate the effect of a very strong confinement effect given by the irreversible adsorption of the Janus particles at the air-water interface. We show that the slowing down of the degrees of freedom related to the rotational diffusion is an efficient way to enhance the directional motion of self propelled Janus colloids.

\section{Experimental Section}

Janus colloids dispersions. Silica beads (Microparticles $\mathrm{GmbH}$, radius $\mathrm{R}=1.06 \pm 0.03 \mu \mathrm{m}$, zeta potential $=-13 \mathrm{mV}$ ) were cleaned by centrifugation cycles using ultra pure water. As described by Love et al. $^{23}$, a monolayer of silica beads was prepared on a silicon wafer by 
drop-casting. $10 \mathrm{~nm}$ thick Titanium and $20 \mathrm{~nm}$ thick Platinum layers were then deposited on surface by sputtering (Pt layer thickness $=20 \mathrm{~nm}$ ). After sonication for 30 minutes silica beads half covered by Pt were dispersed into ultra pure water. Dilute suspensions were cleaned by centrifugation cycles using ultra pure water.

Deposition protocol. Water suspension of Janus particles was sprayed from about $70 \mathrm{~cm}$ distance onto the water surface in a small glass container $\left(1.1 \mathrm{~cm}^{2}\right.$ area) by using an airbrush with a $0.7 \mathrm{~mm}$ nozzle operating at a pressure of $50-100 \mathrm{kPa}$. The depth of water subphase was set at $1.3 \mathrm{~mm}$ to minimize convection and further decrease drift at the water surface. We got isolated particles at interface with an approximate density of 50 particles $\mathrm{mm}^{-2}$. This interfacial density corresponds to a very dilute regime where interaction between colloids can be neglected.

In situ contact angle. The contact angle of single colloids at the interface was measured at the air-water interface by optical microscopy and by a gel trapping method. We measure directly the immersion of colloids at the water interface by imaging from side the water surface as described by Hórvölgyi et $a .^{24}$. Water suspension of colloids was placed between two parallel glass microscope slides and particles attached to the water interface were imaged (see Figure 1(C)). Values reported in the following are obtained by measuring three times the immersion of 5 particles.

For the gel trapping method, ${ }^{25}$ a 2 wt $\%$ Gellen (Phytagel, Sigma-Aldrich) solution in Millipore water was prepared by heating at $90^{\circ} \mathrm{C}$ under magnetic stir. After cooling down to room temperature the gel was formed (at $\mathrm{T}=27-32{ }^{\circ} \mathrm{C}$ ). Beads were spread at the gel surface and again heated at $90^{\circ} \mathrm{C}$ to be trapped at the interface. Immersion depth of beads at gelled water surface is expected to be very similar to the liquid water surface since the two interfaces share almost the same surface tension. Norland Optical Adhesive 81 (NOA81) was poured over the gelled water surface with particles and then photopolymerized by ultraviolet light for 2 minutes. Afterwards the solidified NOA81 layer was peeled off taking particles at 
complementary positions with respect to that at air-gelled water interface. These samples were finally observed by both optical and scanning electron microscopy (SEM). Values of the contact angles by gel trapping reported in the following are the average of more than 20 beads.

Sessile drop contact angles and interfacial tensions. Advancing contact angle of water drops on solid substrates and interfacial tensions were measured by a profile analyzer tensiometer (PAT, Sinterface, Germany). Sessile drop contact angles have been used to evaluate the surface properties of the faces of the Janus colloids, whilst interfacial tensions revealed the purity of the liquids used.

Particle Tracking. Tracking for isolated particle was achieved by using a Basler Scout CCD camera equipped Leica inverted microscope mounted on a Melles Griot optical table and two Leica objectives $(\times 10$ and $\times 32)$. Two types of videos $(1024 \times 768$ pixels $)$ with different field of view approximate $477 \mu \mathrm{m} \times 358 \mu \mathrm{m}$ and $125 \mu \mathrm{m} \times 94 \mu \mathrm{m}$ were recorded at a rate of 30 frames per second. Videos obtained with the larger field of view allow to study particle trajectories at distances much longer than for typical Brownian motion. In order to look into the detailed behavior of Pt catalytic surface and estimate particle's dynamics, the smaller field of view was used. Tracking was performed under Labview (National Instruments) using an image correlation-based approach in order to obtain particle trajectories [time $(\mathrm{t} / \mathrm{s}), \mathrm{x}(\mu \mathrm{m}), \mathrm{y}$ $(\mu \mathrm{m})]$. The software "Stat Tracker St. Andrews" developed by Graham Milne on LabView was used to evaluate the center of mass of the particle with an accuracy of about $0.2 \mu \mathrm{m}$ at a $\times 10$ magnification. By tracking particles using a camera equipped microscope we obtained a sequence of images with a resolution of $\sim 0.466 \mu \mathrm{m} /$ pixel. At the same time image sequence treatment can further improve its resolution and displacement of $0.2 \mu \mathrm{m}$ can be accurately measured.

Particles were tracked first in the absence of $\mathrm{H}_{2} \mathrm{O}_{2}$. Only when particles underwent typical thermal Brownian motion showing negligible drift, we continued the experiments. 
Afterwards, hydrogen peroxide solution was added to water by a microsyringe up to the desired $\mathrm{H}_{2} \mathrm{O}_{2}$ concentration. About 40 minutes elapsed before starting tracking experiments.

We monitored the speed of the Janus colloids in time, it keeps the same within 3 hours and it slowly decreases during about a day. In fact, the concentration of $\mathrm{H}_{2} \mathrm{O}_{2}$ is hardly affected during the measurement time being the interfacial regimes extremely diluted with few particles populate the interface and negligible amount of particles in bulk.

We used an aqueous solution of $\mathrm{H}_{2} \mathrm{O}_{2}$ (30 wt \%, Sigma-Aldrich) and added the solution drop by drop into the system by using micro syringe at different places. A cover is also used to close the container and avoid any exchange with the atmosphere. In this condition $\mathrm{H}_{2} \mathrm{O}_{2}$ is well dissolved in the liquid phase given the high self-diffusion coefficient ${ }^{26}$ of $\mathrm{H}_{2} \mathrm{O}_{2}$ and the relatively high concentration given by the addition of the $\mathrm{H}_{2} \mathrm{O}_{2}$ solution.

To rule out any possible effect of inhomogeneous distribution of $\mathrm{H}_{2} \mathrm{O}_{2}$, we also performed few experiments where we first prepared the solution at a given $\mathrm{H}_{2} \mathrm{O}_{2}$ concentration and then sprayed the particles. Those results agreed with the ones obtained by adding $\mathrm{H}_{2} \mathrm{O}_{2}$ solution drop by drop after spraying of the particles.

\section{Results}

\section{A. Immersion and Orientation of Janus colloids}

\subsection{Trapping the catalytic Janus colloids at the water surface}

We fabricated $\mathrm{Pt}$-half coated colloids from silica beads (Microparticles $\mathrm{GmbH}$, radius $\mathrm{R}=1.06 \pm 0.03 \mu \mathrm{m})$ as described by Love et al. ${ }^{23}$ In Figure 1(A), a scanning electron microscopy (SEM) image shows a Pt-coated colloid displaying the two distinct silica and platinum regions $(\mathrm{Pt}$ layer thickness $=20 \mathrm{~nm}$ ) characteristic of a Janus type particle (see Figure S1 in Supplementary Information). Figure 1(B) sketches the deposition method to trap Pt-coated colloids at the air-water interface. The deposition is obtained by spraying an 
aqueous colloid dispersion, thus avoiding the use of spreading solvents which might contaminate the water surface.

The immersion of colloids at the water interface was measured by optical microscopy as described by Hórvölgyi et al. ${ }^{24}$ (see Figure 1). We calculated a contact angle $\alpha=58 \pm 9^{\circ}$. The latter value is comparable to the contact angle of bare silica colloids, which show $\alpha_{S} \approx 65^{\circ}$.

We have also performed contact angle experiments by a gel trapping method (see Figure S2 Supplementary Information). ${ }^{25}$ As for the optical microscopy method, the contact angle of silica colloids $\alpha_{S}=70.9^{\circ} \pm 3.6^{\circ}$ is slightly larger than the Janus one $\alpha=63.6^{\circ} \pm 2.2^{\circ}$. Contact angle values by gel trapping are somewhat higher than the ones obtained by optical microscopy, which can be ascribed to the slightly different properties of gelled water, the deposition protocol and the temperature at which the trapping occurs.

Note that once attached onto the surface, colloids are trapped at the interface. They cannot escape from the interfacial plane (xy-plane) and transfer to the bulk water, being the transfer energy $\Delta \mathrm{E}=\gamma \mathrm{R}^{2}(1-\cos \alpha)^{2} \approx 4 \times 10^{6} \mathrm{kT}$ much larger than the thermal energy (where $\gamma$ is the surface tension). ${ }^{27}$ When not trapped at the interface, colloids eventually sediment to the bottom of the liquid container. This vertical motion is clearly observed by monitoring focus differences of the microscope images. For the interfacial measurements reported in the following we did not notice any change of the focus with time, which is an additional proof that those particles are confined in the interfacial plane.

\section{Figure 1}

\subsection{Orientation of the Janus colloids at the water surface and surface properties}


The orientation of the Pt cap at the interface is an important parameter for the active motion at the surface. As sketched in Fig. 1(d) an angle $\beta$ can be defined between the interface normal and the Pt cap. Note that for $\beta=0$ or $180^{\circ}$ the $\mathrm{Pt}$ face points to the interface normal and no propulsion is expected in the direction parallel to the interfacial plane.

In order to learn about the possible interfacial orientations, we monitored the orientation of several Janus colloids by a gel trapping procedure. By using Leica inverted microscope with a $\times 100$ oil phase Leica objective and due to the refractive index difference between $\mathrm{Pt}$ and silica, we classified the orientation of Janus particles with respect to the interface. ${ }^{28}$ Figure 2 display an optical image together with the distribution of orientation. Three classes of orientation are distinguished: (i) when the Janus boundary is parallel to the air water interface the colloids appear either completely dark, when the Pt face is immersed in water, or completely white when the silica face is immersed in water. When the Janus boundary is not parallel to interface both black and white domains are clearly distinguished on the particle. Black region of the colloid is the Pt-coated surface. For most of the particle, the Pt cap is partially immersed in water. More precisely, $70 \%$ out of 250 colloids have the Janus boundaries not parallel to the air-water interfacial plane. For $20 \%$ of the particles the Pt face is completely immersed in water $\left(\beta=0 \pm 30^{\circ}\right)$ while only less than $10 \%$ of the particle have their silica face completely immersed. .

To evaluate the surface energy of the platinum and silica surfaces, we also prepared planar silica and platinum coated substrates and measured the contact angles of water drops. Sessile drop contact angles are: $42 \pm 2^{\circ}$ (silica) and $39 \pm 2^{\circ}$ (platinum). Those values point to a small energy difference between different orientation qualitatively consistent with the results shown in Figure 2 and confirm the random orientation as observed for Au-PS particles at the oil-water interface. ${ }^{28}$ Note that difference between the contact angles of millimetric drops and 
micron sized colloids could be explained in terms of the different interaction length scales and fabrication protocols. $^{29}$

\section{Figure 2}

\section{B. Active motion and rotational diffusion}

\subsection{Active velocity of Janus particle at the air-water interface}

Figure 3 displays two dimensional trajectories for Janus colloids at the water surface under different $\mathrm{H}_{2} \mathrm{O}_{2}$ fuel concentrations in a field of view of $477 \mu \mathrm{m} \times 358 \mu \mathrm{m}$. In the absence of $\mathrm{H}_{2} \mathrm{O}_{2}$, particles undergo characteristic Brownian motion confining their trajectories in small areas (end-to-end distances of trajectories remain below $4 \mu \mathrm{m}$ lengths during $20 \mathrm{~s}$ ). In presence of $\mathrm{H}_{2} \mathrm{O}_{2}$, directional displacements in the order of $100 \mu \mathrm{m}$ have been measured over an observation time of 20 seconds. Note that even for such long distances $(\approx 400$ times the particle radius) and times only few directional changes are observed. In contrast, in the bulk clear long range random walks have been previously observed for Pt-coated polystyrene beads in bulk water $\left(\tau_{\mathrm{R}}=1 / D_{R} \approx 6 \mathrm{~s}\right.$ and $V \approx 6 \mu \mathrm{m} / \mathrm{s}$, in the bulk). ${ }^{17,22}$ At longer times and distances, the possibility of very long range random walks might exist. However, those scales are beyond the experimental windows of particle tracking video microscopy shown here.

\section{Figure 3.}

To evaluate the active velocity of the Janus particles at the air-water interface we tracked particles in a field of view $125 \mu \mathrm{m} \times 94 \mu \mathrm{m}$. The mean squared displacement $(M S D)$ is presented in Figure 4(A) as a function of the delay time for $\mathrm{H}_{2} \mathrm{O}_{2}$ concentrations in the range between 0 and $6 \mathrm{v} / \mathrm{v} \%$. In absence of $\mathrm{H}_{2} \mathrm{O}_{2}, M S D$ is linear as expected for random Brownian motion. In the presence of $\mathrm{H}_{2} \mathrm{O}_{2}$, all $M S D$ are parabola. The general expression for the twodimensional projection of the $M S D$ for active colloids dynamics in the bulk reads: ${ }^{30}$ 
$M S D(\Delta t)=4 D_{T, 0} \Delta t+\frac{V^{2} \tau_{R}^{2}}{3}\left[\frac{2 \Delta t}{\tau_{R}}+\exp \left(-\frac{2 \Delta t}{\tau_{R}}\right)-1\right]$,

which reduces to :

$M S D=4 D_{T, 0} \Delta \mathrm{t}+2 / 3 V^{2} \Delta \mathrm{t}^{2}$

when the rotational diffusion time $\tau_{\mathrm{R}}$ is much larger than $\Delta \mathrm{t}$.

In equations 2 and $3, D_{T, 0}$ is the translational Brownian diffusion coefficient.

At the air-water interface, the parameters of equation 2 and 3 are different from the bulk values since the colloid is just partially immersed in the bulk liquid. We determined $D_{T, 0}$ with the data for $\left[\mathrm{H}_{2} \mathrm{O}_{2}\right]_{\mathrm{V}}=0 \%$ and constrained the value of $D_{T, 0}$ for all other fits (Figure $3(\mathrm{~A})$ ). We found a slowing down of $D_{T, 0}$ at the interface when compared to the theory ${ }^{31}$ and a value very similar to the bulk value (which we will discuss in section 4).

Note that equation 3 , assuming $\tau_{\mathrm{R}} \gg>\Delta$, leads to very good fits to the experimental data. In Figure 4(B) the velocity $V$ (averaged for 8 particles for each concentration) increases with the fuel concentration and for $\left[\mathrm{H}_{2} \mathrm{O}_{2}\right]_{\mathrm{V}}=6 \%$ the average velocity is ca. $18 \mu \mathrm{m} \mathrm{s}^{-1}$, which is about twice the velocity measured in the bulk (see Figure S3 and S4 in Supplementary Information).

\section{Figure 4.}

To check the robustness of our results, we also performed a series of control experiments in order to rule out the effect of drift due to convection, which is always to be suspected at the surface. ${ }^{32}$ We measured the drift velocities by studying the diffusion of passive silica beads for the same fuel concentrations used for Janus particles. As shown by the filled squares in Figure 4(B), velocities around $1 \mu \mathrm{m} \mathrm{s}^{-1}$ have been measured, which are much smaller than the velocities measured in active conditions. 
For some trajectories we measured, by performing image analysis it was also possible to achieve additional information on the position of the platinum coverage during the motion. An example of trajectory of an active colloid moving at the water surface is shown in Figure 5. The images below the trajectory (red line) in Figure 5 are real images of the Janus particle, which show a darker region which is the coated Pt layer. To enhance the contrast between the two regions, images were binarized using a constant threshold (by implementing a routine in $I D L$ software). The resulting images are shown in the insets above the trajectory (Figure 5). White color in the thresholded images corresponds to the darker part of the colloid in the raw images and it represents the Pt region. The orientation of the Pt layer normal is the same as the moving direction with the Janus particle moving with the platinum segment in the rear. The same observation was done for all beads where the Pt area could be detected. Hence, it demonstrates that the catalytic layer drives the particle to move directionally; ruling out that drift is causing the directional motion. Moreover, it confirms that the motion occurs in the direction opposite to the catalytic site.

Within the field of view used in Figure $5(125 \mu \mathrm{m} \times 94 \mu \mathrm{m})$, no significant directional changes of the trajectories were observed. Imaging approximately constant thresholded areas agrees with the expected slowing down of rotations about an axis perpendicular to the interface normal (x- and $\mathrm{y}$-axis). ${ }^{33}$

Figure 5.

\subsection{Rotational diffusion times}

In this section, we attempt evaluating the characteristic times, which describe the occurrence of change of direction during the active motion of Janus particles at the interface.

We calculated the autocorrelation function of the velocity vector $v$, $\langle v(\Delta t) \cdot v(0)\rangle$, of the trajectories shown in Figure 3 (see Figure S4 in Supplementary Information). 
The quantity $\langle v(\Delta t) \cdot v(0)\rangle$ describes the probability of a colloid to keep the same velocity after a delay time $\Delta \mathrm{t}$. This probability is lost when the colloid either changes significantly its direction or its velocity modulus. For active colloids in bulk the autocorrelation function of the velocity can be written as: ${ }^{34}$

$$
\left\langle v(\Delta \mathrm{t}) \cdot v(0)>=4 D_{T, 0} \delta(\Delta \mathrm{t})+V^{2} \exp \left(-D_{R} \Delta \mathrm{t}\right)\right.
$$

where $4 D_{T, 0} \delta(\Delta \mathrm{t})$ corresponds to the passive Brownian motion contribution to the autocorrelation and $V$ is the active velocity. ${ }^{16,34}$ For relatively high $V$, the Brownian contribution to the autocorrelation is negligible (see Figure S4 in Supplementary Information). By fitting $\langle v(\Delta \mathrm{t}) \cdot v(0)\rangle=V^{2} \exp \left(-D_{R} \Delta \mathrm{t}\right)$, we obtained $D_{R}$ and the active velocity $V$ (see Figure S4 in Supplementary Information). Autocorrelation function of the velocity vector $v$ could be also fitted by accounting for an active term written as in the runand-tumble model, $\langle\boldsymbol{v}(\Delta \mathrm{t}) \cdot \boldsymbol{v}(0)\rangle=V^{2}\left(\tau_{\mathrm{v}}-\Delta \mathrm{t}\right) / \tau_{\mathrm{v}}$, where $\tau_{\mathrm{v}}$ is the characteristic time at which the directional change occurs (see Figure S4 in Supplementary Information). ${ }^{16}$ These two different fits lead to very similar results.

We plotted $\tau_{\mathrm{R}}=1 / D_{R}$ in Figure 6. A comparison with the rotational diffusion time of a colloid immersed in the bulk water $\left(8 \pi \eta \mathrm{R}^{3} / \mathrm{kT}\right.$, where $\eta$ is the water viscosity and $\mathrm{kT}$ the thermal energy, valid for no-slip condition) or half immersed at the air-water interface $\left(4 \pi \eta \mathrm{R}^{3} / \mathrm{kT}\right)$ and rotating about the $\mathrm{z}$-axis (parallel to the interface normal) is also shown in the same figure. $^{33}$

Since the contact angle of the Janus colloids at the water surface is in between 0 and $90^{\circ}$, rotational diffusion times should also be in between the values for totally and half-immersed particles. However in presence of $\mathrm{H}_{2} \mathrm{O}_{2}$, some of the data show relatively high values of the rotational diffusion time, which can be even larger than the rotational diffusion time of a colloid completely immersed in the bulk water $\left(8 \pi \eta R^{3} / k T\right)$. 
Finally, a persistence length $\ell=V / D_{R} \approx 140 \mu \mathrm{m}$ can be estimated, which is more than ten times the value obtained in the bulk at the same $\mathrm{H}_{2} \mathrm{O}_{2}$ concentration (see Figure 7; in the bulk: $\left[\mathrm{H}_{2} \mathrm{O}_{2}\right]_{\mathrm{V}}=5 \%, V \approx 9 \mu \mathrm{m} / \mathrm{s}$ and $\tau_{\mathrm{R}} \approx 1 \mathrm{~s}$, see also Figure $\mathrm{S} 4$ in Supplementary Information). ${ }^{17,22}$

\section{Figure 6.}

Figure 7.

\subsection{Correlation between velocity and directional changes of the trajectory}

In order to analyze the features of the trajectories displayed in Figure 3 we have also computed the autocorrelation function $\langle\mathrm{v}(\Delta \mathrm{t}) \cdot \mathrm{v}(0)\rangle$ of the velocity modulus, $\mathrm{v}=\sqrt{v_{x}^{2}+v_{y}^{2}}$ (where $\mathrm{v}_{\mathrm{x}}$ and $\mathrm{v}_{\mathrm{y}}$ are the components of the velocity in the interfacial plane).

$\langle\mathrm{v}(\Delta \mathrm{t}) \cdot \mathrm{v}(0)\rangle$ can be related to the orientation of the out of plane orientation of Pt cap as described by the angle $\beta$ sketched in Fig.1(d). For most of the trajectories we analyzed $\langle\mathrm{v}(\Delta \mathrm{t}) \cdot \mathrm{v}(0)\rangle$ keeps constant when the delay time increases; and directional changes occur smoothly with the Janus colloid rotating only about the interface normal. Figure 8 shows a typical trajectory and the autocorrelation function of the velocity modulus together with the autocorrelation function of the velocity vector. The decay of the latter can be then ascribed to directional changes within the interfacial plane while the velocity modulus keeps approximately constant.

Figure 8. 
A different scenario occurs for few trajectories when a sharp directional change occurs. In this case, we could also observe a significant change of the angle $\beta$ which defines the orientation of the platinum face of the particle with respect to the interface. Indeed, a clear correlation between the sharp directional change of the trajectory, the change of orientation of the platinum region can be observed in Figure 9. Far from the turning point, from the thresholded images one could distinguish two different regions which are the silica and the platinum faces of the Janus colloid. Close to the turning point, these two separated regions can not be clearly detected. Note that the autocorrelation function of the velocity modulus is not constant but decreases with $\Delta \mathrm{t}$. By fitting the autocorrelation function of the velocity vector $\langle v(\Delta \mathrm{t}) \cdot v(0)\rangle$ as before, we find a rotational time $\tau_{\mathrm{R}}=1 / D_{R} \approx 5 \mathrm{~s}$ whilst from the decrease of $\langle\mathrm{v}(\Delta \mathrm{t}) \cdot \mathrm{v}(0)\rangle$ a characteristic time of about $17 \mathrm{~s}$ reveals the dramatic slowing down of the out of plane rotational diffusion.

These findings indicate that the platinum face changes its orientation at the sharp turning point, rotating about both an axis parallel ( $\mathrm{x}$ - and $\mathrm{y}$ - axis) and perpendicular to the interface normal (z-axis). The two rotations are related to the in-plane and out of plane orientations of the platinum face at the interface respectively as can be observed in Figure 9.

\section{Figure 9.}

\section{Discussion}

We can now discuss the results reported in the previous sections describing the directional motion of Janus colloids at the air-water interface. Comparing to previous investigations, ${ }^{17,22}$ we measured velocity as high as $18 \mu \mathrm{m} / \mathrm{s}$ (twice the value measured in the bulk) and directional trajectories, which show only few directional changes (Figure 3). 
To explain why directional trajectories are much more persistent at the surface with respect to the bulk, we need to recall that the randomization process is dictated by the rotational diffusion time. A self-propelled colloid in the bulk can experience rotation about three different Cartesian axes with the same probability and a single diffusion rotational time $\tau_{\mathrm{R}} \approx 1 / D_{R}$. In contrast at the interface (xy-plane) for Janus particles, three different rotational times might exist. Only one rotation $D_{R, z}$ (about an axis parallel to the interfacial normal) would be allowed in the ideal no-slip condition (see Figure 4 and 6). As known from partial wetting dynamics, $D_{R, x}$ and $D_{R, y}$ (rotational diffusion about the x- and y-axis respectively) are significantly slowed down (but not completely suppressed) because of the high dissipation occurring close to the triple line..$^{35,36}$

By calculating the autocorrelation function of the velocity vector and modulus, we also gain information on a slowing down of the rotational diffusion (Figure 6, 8, 9). Some rotational times are comparable or even higher than the bulk values for spheres. To fit a rotational time of $\approx 10$ seconds (Figure 6 ) using the free rotational diffusion time of $2 \mathrm{D}$ platelet $1 / D_{R, z}=$ $32 \eta \mathrm{R}^{3} / 3 \mathrm{kT}$, would result in an equivalent radius $\mathrm{R}=1.56 \mu \mathrm{m}(50 \%$ larger than the colloid radius). ${ }^{37}$

As reported in the literature, the slowing down of the translational and rotational diffusion of colloids at the interface is a rather complex issue. ${ }^{38,39,40}$ A purely hydrodynamic picture of the problem may relate the diffusion only to the contact angle of the particle (and the viscosities). ${ }^{31}$ However many experimental data in the literature reported deviations from hydrodynamic predictions. ${ }^{38,39,40}$ This slowing down may be attributed to an interfacial friction which adds to the hydrodynamic friction. In particular, thermal fluctuations of the contact line over the colloid surface can slow down significantly the interfacial motion. ${ }^{35}$ Very recently Boniello et al. reported a systematic investigation on the slowing down of translational and rotational diffusion of spherical and ellipsoidal particles at the interface. ${ }^{41}$ 
The slowing down can be modeled as an extra friction due to the contact line fluctuations. This interfacial friction could be quantified for two different cases. The first one is related to a fluctuating contact line around and an equilibrium position as in the Blake's model of partial wetting. ${ }^{36}$ The second scenario deals with the friction resulting from thermal capillary wave on a pinned contact line.

Here we have demonstrated that by confining the motion of self-propelled particles at the water surface we are able to enhance motion directionality by reducing the particle rotational motion.

We also observed that Janus particles propel away from the catalytic site, which agrees with a nanobubble detachment mechanism proposed by Gibbs and Zhao for the same silica-Pt colloids used here (Figure 5). ${ }^{22}$ Still the direction of motion alone is not enough to determine the mechanism of propulsion; and nanobubbles cannot be observed in the resolution of optical microscopy. Recently, self-diffusiophoresis and self-electrophoresis have been proposed as alternative mechanisms for the propulsion of polystyrene-Pt colloids. ${ }^{42,21}$ However, those mechanisms are not able to explain the scaling of the propulsion velocity with the square of the surface tension of the aqueous solution as observed for silica-Pt colloids. ${ }^{22}$

An additional major difference between silica-Pt and polystyrene-Pt colloids fabricated so far consists in the thickness of the platinum layer. The thickness used in the latter colloids is about $10 \mathrm{~nm}$ or lower. ${ }^{2120}$ Whilst, a thickness of $20 \mathrm{~nm}$ or higher was used for silica-Pt colloids. ${ }^{22}$ This thickness affects strongly the decomposition rate of $\mathrm{H}_{2} \mathrm{O}_{2}$; and for thickness lower than $10 \mathrm{~nm}$ the decomposition rate decreases significantly, affecting the propulsion mechanism.$^{2021}$

Independently from the detailed knowledge of the propulsion mechanism, one can always define an effective propulsion force. ${ }^{43}$ Here, the propulsion force of the Janus colloid depends on the angle $\beta$ between the interface normal and the platinum cap (see Figure 1(d)). As suggested from Figure 5, $\beta$ might lay in between $45^{\circ}$ and $90^{\circ}$ during directional 
trajectories, which reduces the propulsion force $F_{P}$ by a factor $\sin (\beta)$. Nonetheless the active velocity at the interface is higher than in the bulk. One might attribute this enhancement to a lower translational friction coefficient, since the particle is partially immersed in water and in air, which has a negligible viscosity. For the contact angle we measured, the interfacial friction is predicted to be $\approx 0.73$ the bulk value $6 \pi \eta \mathrm{R} .{ }^{31}$ However as pointed out before also the translational friction is increased at the interface because of an extra contact line friction not present in the bulk. The discrepancy between active velocity at the interface and in the bulk might be due to the fact that bulk velocities have been measured close to the solid wall of the bottom of the glass container at gap distances shorter than the particle size. ${ }^{34}$ In this case, the hydrodynamic friction would be much higher than in the bulk. If the colloid is moving at the solid surface the translational friction would be $\approx 56 \%$ higher than in the bulk. ${ }^{44,45}$

\section{Conclusion}

We have demonstrated that by trapping Janus colloids at the water surface we are able to enhance their directional active motion. We measured long range directional trajectories at the water surface with persistence length $\ell=$ ca. $140 \mu \mathrm{m}$. We explained these findings by the slowing down of the particle rotational diffusion, which is the main limiting factor of directional active motion. Surprisingly, we measured a velocity of propulsion, which is significantly higher than the bulk one at the same $\mathrm{H}_{2} \mathrm{O}_{2}$ concentration.

A general strategy can be envisioned in the future for an improved performance of active colloids. In principle, two dimensional or one dimensional confinement effects can be used to perform tasks such as transport cargos and degrading water contaminants in a very efficient way. 


\section{Acknowledgements}

We thank Paolo Malgaretti, Aidan Brown and Wilson Poon for discussion. We acknowledge financial support from Labex Numev, Labex CheMISyst and the Scientific Council of the University of Montpellier. A. S. gratefully acknowledges the Alexander von Humboldt foundation for a research fellowship.

\section{REFERENCES}

1 W. F. Paxton, K. C. Kistler, C. C. Olmeda, A. Sen, S. K. St Angelo, Y. Cao, T. E. Mallouk, P. E. Lammert and V. H. Crespi, J. Am. Chem. Soc., 2004, 126, 13424-31.

2 P. Dhar, T. M. Fischer, Y. Wang, T. E. Mallouk, W. F. Paxton and a Sen, Nano Lett., 2006, 6, 66-72.

3 R. Dreyfus, J. Baudry, M. L. Roper, M. Fermigier, H. A. Stone and J. Bibette, Nature, 2005, 437, 862-865.

$4 \quad$ H.-R. Jiang, N. Yoshinaga and M. Sano, Phys. Rev. Lett., 2010, 105, 268302.

$5 \quad$ S. J. Ebbens and J. R. Howse, Soft Matter, 2010, 6, 726.

6 D. Kagan, R. Laocharoensuk, M. Zimmerman, C. Clawson, S. Balasubramanian, D. Kang, D. Bishop, S. Sattayasamitsathit, L. Zhang and J. Wang, Small, 2010, 6, 2741-7.

7 F. Kümmel, B. ten Hagen, R. Wittkowski, I. Buttinoni, R. Eichhorn, G. Volpe, H. Löwen and C. Bechinger, Phys. Rev. Lett., 2013, 110, 198302.

8 L. Baraban, M. Tasinkevych, M. N. Popescu, S. Sanchez, S. Dietrich and O. G. Schmidt, Soft Matter, 2012, 8, 48.

9 J. Palacci, S. Sacanna, A. Vatchinsky, P. M. Chaikin and D. J. Pine, J. Am. Chem. Soc., 2013, 135, 15978-15981.

10 L. Soler, V. Magdanz, V. M. Fomin, S. Sanchez and O. G. Schmidt, ACS Nano, 2013, 7, 9611-20.

11 B. Jurado-Sánchez, S. Sattayasamitsathit, W. Gao, L. Santos, Y. Fedorak, V. V Singh, J. Orozco, M. Galarnyk and J. Wang, Small, 2014, 11, 499-506.

12 P. Tierno, R. Albalat and F. Sagués, Small, 2010, 6, 1749-52.

13 S. Gangwal, O. Cayre, M. Bazant and O. Velev, Phys. Rev. Lett., 2008, 100, 058302.

14 S. Saha, R. Golestanian and S. Ramaswamy, Phys. Rev. E, 2014, 89, 062316. 
15 B. El Pinchasik, H. Möhwald and A. G. Skirtach, Small, 2014, 10, 2670-2677.

16 Z. Wang, H.-Y. Chen, Y.-J. Sheng and H.-K. Tsao, Soft Matter, 2014, 10, 3209-17.

17 J. Howse, R. Jones, A. Ryan, T. Gough, R. Vafabakhsh and R. Golestanian, Phys. Rev. Lett., 2007, 99, 048102.

18 R. Golestanian, T. Liverpool and A. Ajdari, Phys. Rev. Lett., 2005, 94, 1-4.

19 S. Ebbens, M.-H. Tu, J. Howse and R. Golestanian, Phys. Rev. E, 2012, 85, 020401.

20 S. Ebbens, D. Gregory, G. Dunderdale, J. Howse, Y. Ibrahim, T. B. Liverpool and R. Golestanian, Eur. Phys. Lett., 2014, 106, 58003.

21 A. Brown and W. Poon, Soft Matter, 2014, 10, 4016-4027.

22 J. G. Gibbs and Y.-P. Zhao, Appl. Phys. Lett., 2009, 94, 163104.

23 J. C. Love, B. D. Gates, D. B. Wolfe, K. E. Paul and G. M. Whitesides, Nano Lett., 2002, 2, 891-894.

24 Z. Horvolgyi, S. Nemeth and J. H. Fendler, Colloids Surfaces A Physicochem. Eng. Asp., 1993, 71, 327-335.

25 A. Maestro, E. Guzmán, F. Ortega and R. G. Rubio, Curr. Opin. Colloid Interface Sci., 2014, 19, 355-367.

26 S. a M. Van Stroe-Biezen, F. M. Everaerts, L. J. J. Janssen and R. a. Tacken, Anal. Chim. Acta, 1993, 273, 553-560.

27 P. Pieranski, Phys. Rev. Lett., 1980, 45, 569.

28 B. J. Park, T. Brugarolas and D. Lee, Soft Matter, 2011, 7, 6413.

29 A. Stocco, G. Su, M. Nobili, M. In and D. Wang, Soft Matter, 2014, 10, 6999-7007.

30 J. Palacci, C. Cottin-Bizonne, C. Ybert and L. Bocquet, Phys. Rev. Lett., 2010, 105, 088304.

31 T. M. Fischer, P. Dhar and P. Heinig, J. Fluid Mech., 2006, 558, 451.

32 T. Gehring and T. M. Fischer, J. Phys. Chem. C, 2011, 115, 23677-23681.

33 M. E. O’Neill, K. B. Ranger and H. Brenner, Phys. Fluids, 1986, 29, 913.

34 S. Ebbens, R. A. L. Jones, A. J. Ryan, R. Golestanian and J. R. Howse, Phys. Rev. E Stat. Nonlinear, Soft Matter Phys., 2010, 82.

35 D. M. Kaz, R. Mcgorty, M. Mani, M. P. Brenner and V. N. Manoharan, Nat. Mater., 2012, 11, 138-42. 
36 T. D. Blake, J. Colloid Interface Sci., 2006, 299, 1-13.

37 P. Holmqvist, V. Meester, F. Westermeier and D. Kleshchanok, J. Chem. Phys., 2013, 139.

38 K. Du, J. A. Liddle and A. J. Berglund, Langmuir, 2012, 28, 9181-8.

39 A. Stocco, T. Mokhtari, G. Haseloff, A. Erbe and R. Sigel, Phys. Rev. E, 2011, 83, 111 .

40 A. Nelson, D. Wang, K. Koynov and L. Isa, Soft Matter, 2015, 11, 118-129.

41 G. Boniello, C. Blanc, D. Fedorenko, M. Medfai, N. Mbarek, M. In, M. Gross, A. Stocco and M. Nobili, Nat. Mater., 2015, Doi:10.1038/nmat4348.

42 S. J. Ebbens and J. R. Howse, Langmuir, 2011, 27, 12293-6.

43 B. Hagen, R. Wittkowski, D. Takagi, K. Felix, C. Bechinger and L. Hartmut, J. Phys. Condens. Matter, 2015, in press.

44 A. J. Goldmans, R. G. Cox, H. Brenner and O. Neill, Chem. Eng. Sci., 1967, 22, 637.

45 B. S. H. Lee, R. S. Chadwickt and L. G. Leal, J. Fluid Mech., 1979, 93, 705-726. 
Figure 1. (A) SEM image of fabricated Janus colloids. (B) Scheme for depositing particles at air-water interface by airbrush spraying. (C) Microscopy image of a Janus colloid at the airwater interface. (D) Sketch of the contact angle $\alpha$ of the colloid at the air-water interface and the angle $\beta$ defying the orientation of the Pt cap with respect to the interface.
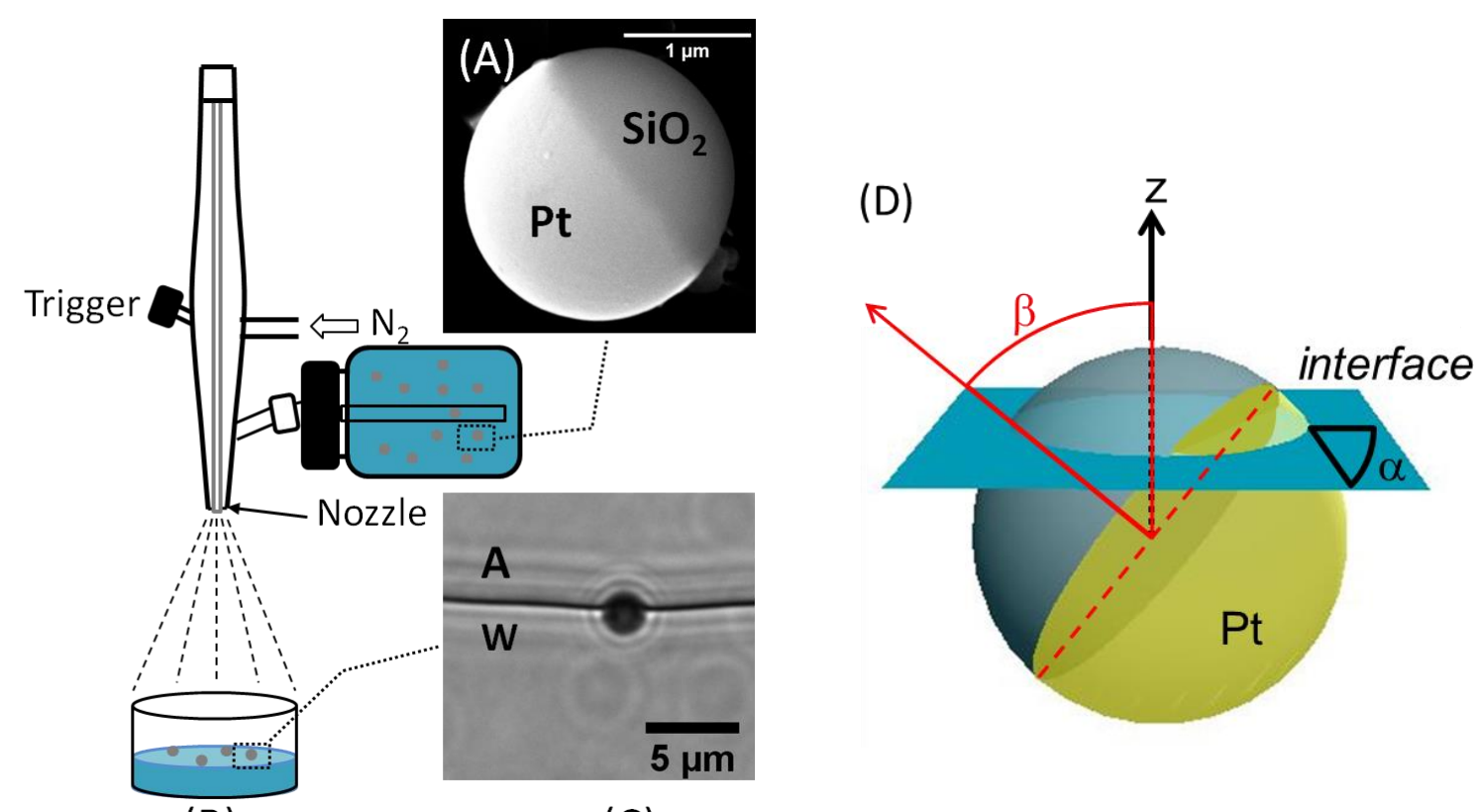

(B)

(C)

Figure 2. (A) Optical microscopy image showing Janus $\mathrm{Pt}-\mathrm{SiO}_{2}$ colloids at interface obtained by a gel trapping method. The visible parts of the particles were in gelled water. Black region represents the Pt-coated surface. (B) Histogram of the particle orientation distribution. The gray, black and white bars represent separately the numbers of particles with Janus boundary rotated out of the interfacial plane (gray), particles with Pt cap immersed in water (black) and particle with Pt-coated surface exposed in air (white).
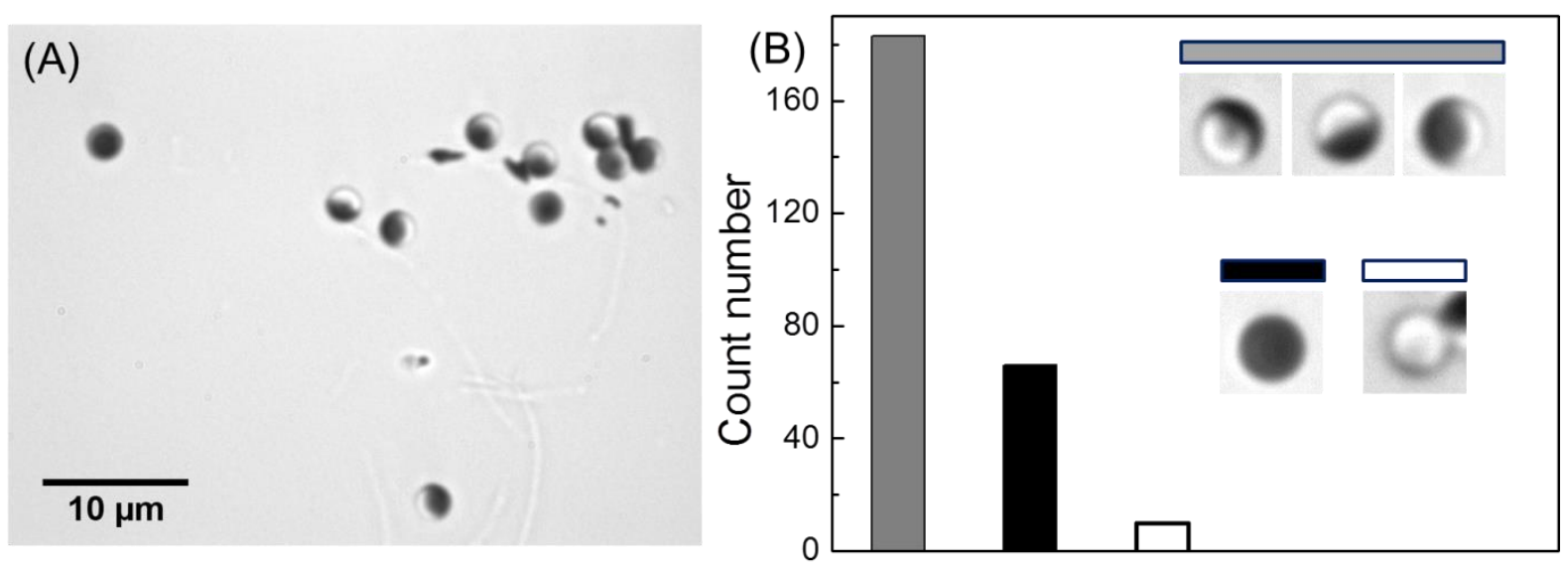
Figure 3. Trajectories of Janus particles at the water interface (xy-plane) under different fuel concentrations $\left[\mathrm{H}_{2} \mathrm{O}_{2}\right]_{\mathrm{V}}$ over 20 seconds. Measurements performed at a field of view of 477 $\mu \mathrm{m} \times 358 \mu \mathrm{m}$.

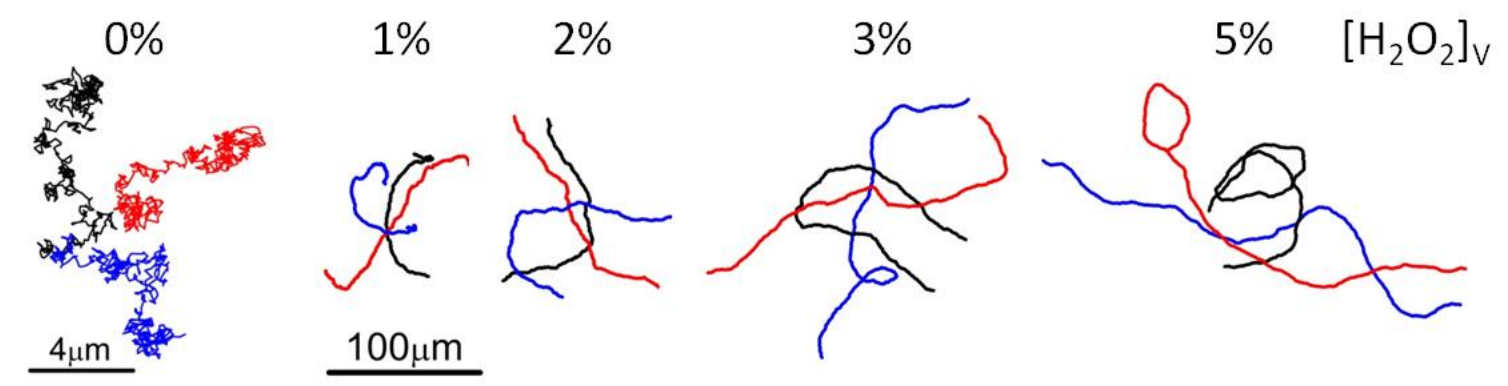


Figure 4. (A) Mean squared displacement $(M S D)$ as a function of the delay time for different $\left[\mathrm{H}_{2} \mathrm{O}_{2}\right]_{\mathrm{V}}$. Solid lines represent best fits of $M S D=4 D_{T, 0} \Delta \mathrm{t}+2 / 3 V^{2} \Delta \mathrm{t}^{2}$. (B) Active velocity $V$ as a function of $\left[\mathrm{H}_{2} \mathrm{O}_{2}\right]_{V}$.
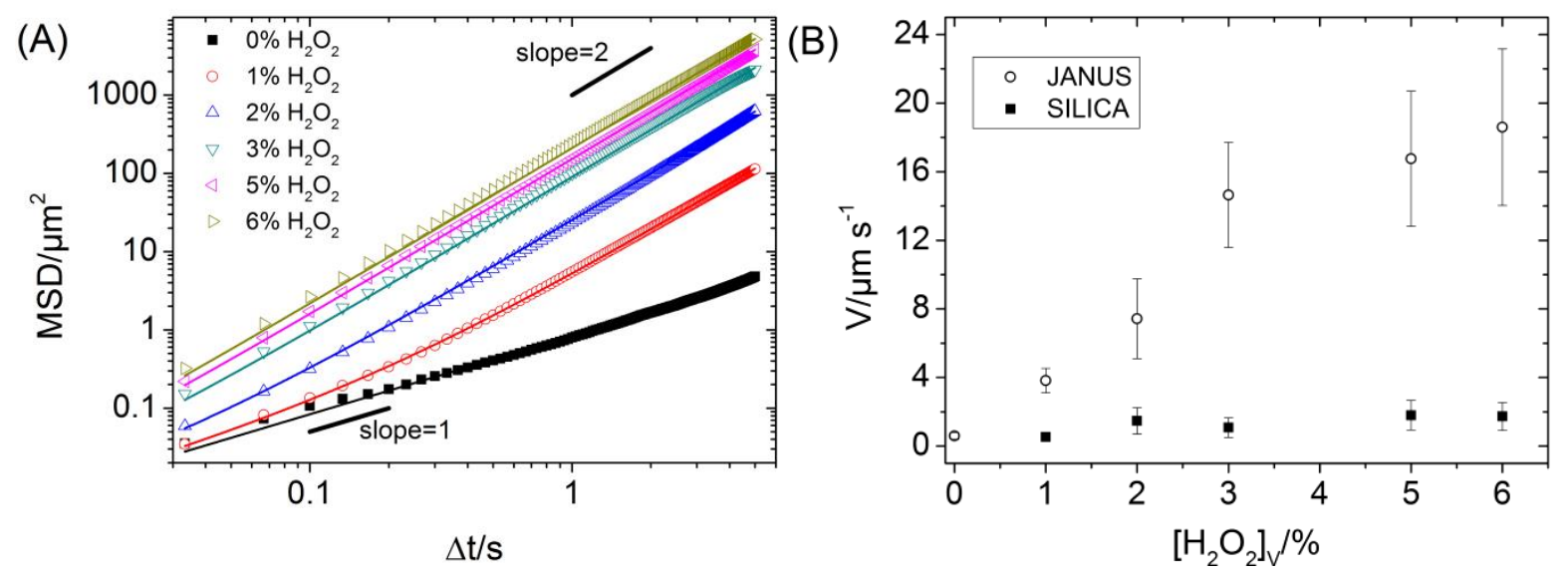

Figure 5. Trajectory (red line) of a Janus colloid at 5\% $\left[\mathrm{H}_{2} \mathrm{O}_{2}\right]_{\mathrm{v}}$ at the water surface. Three pairs of magnified images [raw (below the trajectory) and thresholded (above the trajectory)] are inserted from right to left at time 2, 4, 6 seconds respectively. Measurements performed at a field of view $125 \mu \mathrm{m} \times 94 \mu \mathrm{m}$.

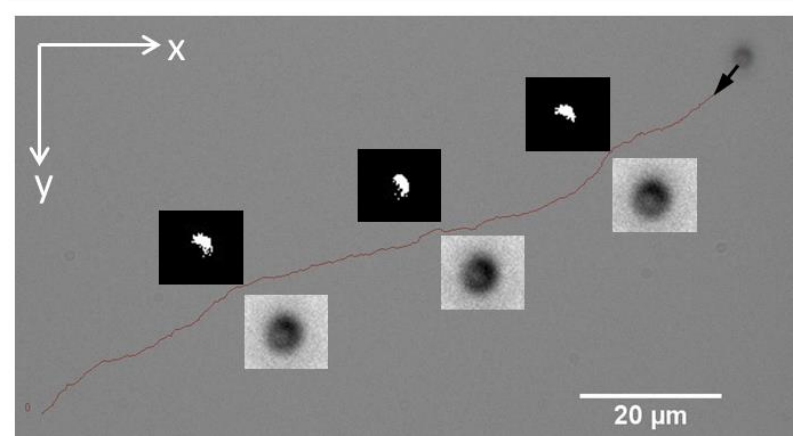


Figure 6. Rotational times extracted from the fit of the velocity autocorrelation function. The solid line corresponds to the rotational diffusion time of a Brownian colloid in water and the dashed line to a half-immerged colloid at the water-air interface.

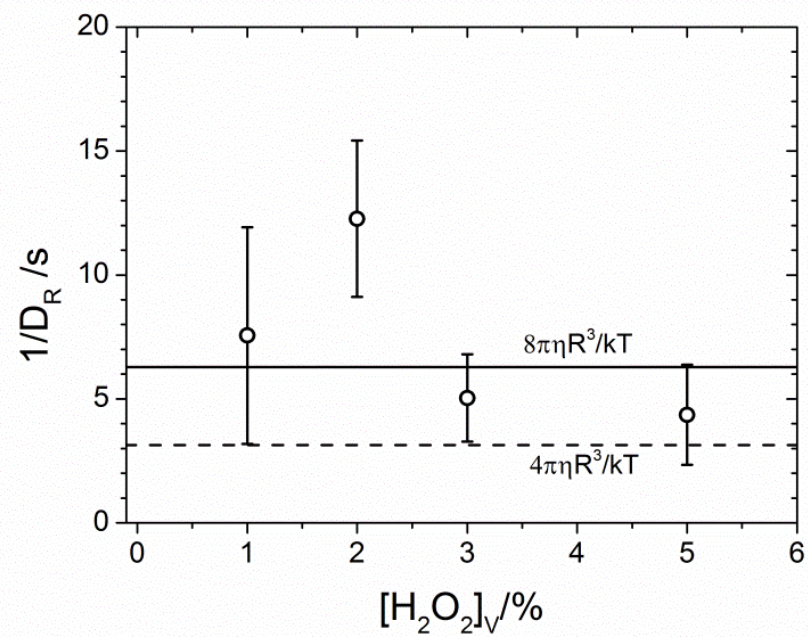

Figure 7. Trajectories of a Janus particle at the water interface and in bulk water at $\left[\mathrm{H}_{2} \mathrm{O}_{2}\right]_{\mathrm{V}}=5 \%$ for $t=16 \mathrm{~s}$.

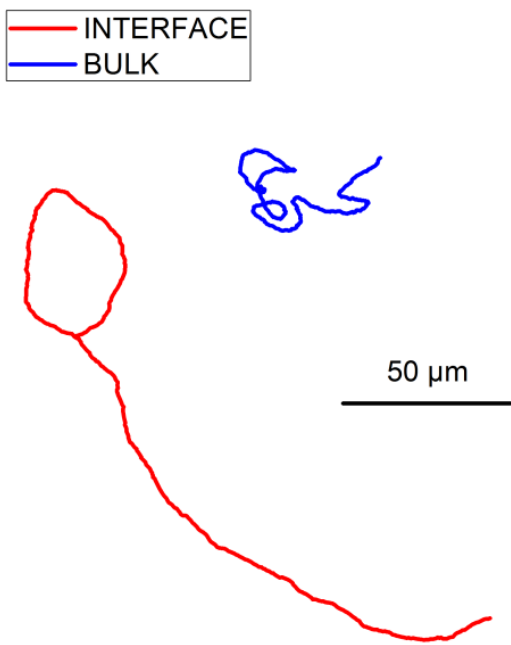


Figure 8. (A) Trajectory of a Janus colloid at the interface from Fig. 3. (B) Autocorrelation functions of the velocity modulus $\langle\mathrm{v}(\Delta \mathrm{t}) \cdot \mathrm{v}(0)\rangle$ and vector $\langle v(\Delta \mathrm{t}) \cdot v(0)\rangle$. Black lines represent the fits of the data.

(A)

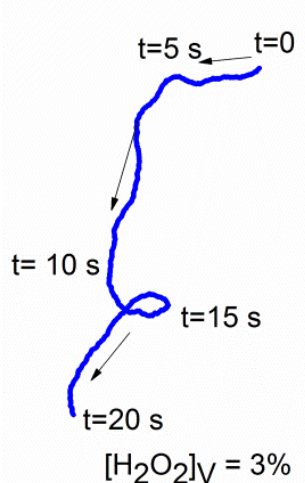

(B)

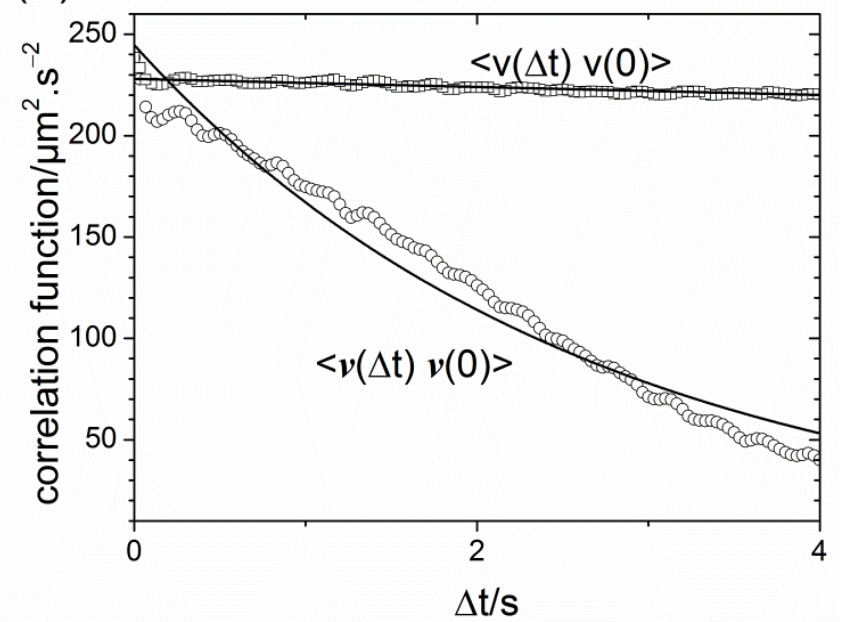

Figure 9. (A) Trajectory (green line) of an active Janus colloid at the water surface. Pairs of magnified images (raw and thresholded) inserted from left to right at different times. (B) Autocorrelation functions of the velocity $\langle\mathrm{v}(\Delta \mathrm{t}) \cdot \mathrm{v}(0)\rangle$ and vector $\langle v(\Delta \mathrm{t}) \cdot v(0)\rangle$. Black lines represent the fits of the data.

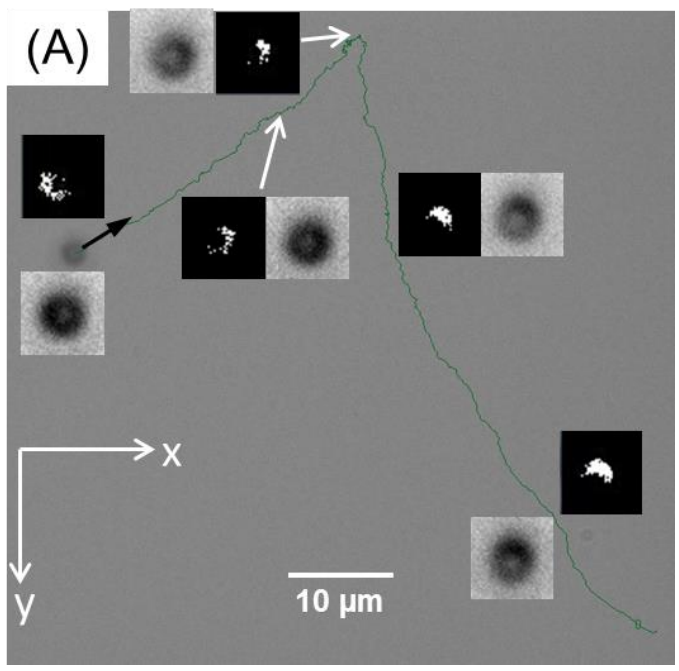

(B)

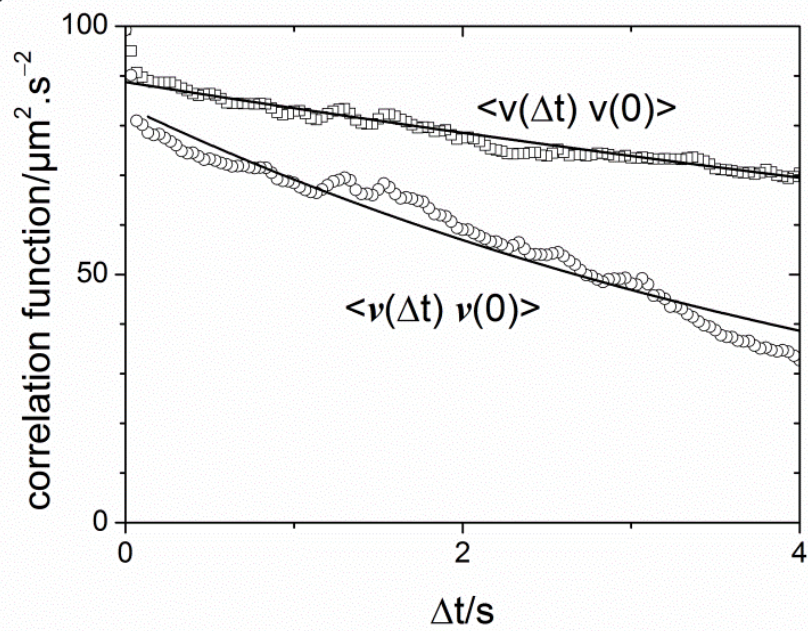


Self propelled Janus colloids show enhanced active motion when trapped at the water surface. Trajectories are more directional and velocities are higher than in the bulk. The confinement effect given by the air-water interface strongly reduces the rotational motion of the colloid while not hindering self-propulsion.

Keyword: self-propulsion, interface, Janus particles, diffusions, partial wetting

X. Wang, M. In, C. Blanc, M. Nobili, A. Stocco*

Enhanced active motion of Janus Colloids at the water surface

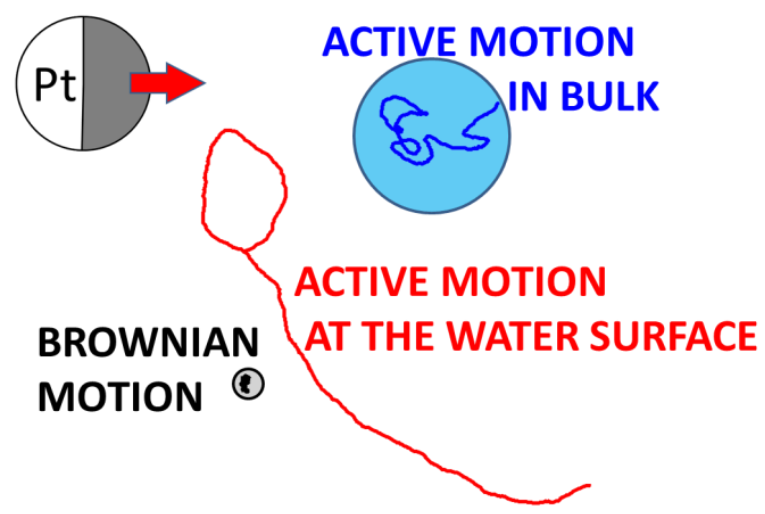




\section{SUPPLEMENTARY INFORMATION}

\section{Enhanced active motion of Janus Colloids at the water surface}

Xiaolu Wang, ${ }^{1}$ Martin In, ${ }^{1}$ Christophe Blanc, ${ }^{1}$ Maurizio Nobili, ${ }^{1}$ Antonio Stocco ${ }^{1,2 *}$

${ }^{1}$ Laboratoire Charles Coulomb (L2C), UMR 5221 CNRS University of Montpellier, Montpellier, F-France.

${ }^{2}$ DWI - Leibniz-Institut für Interaktive Materialien, Aachen D-52056, Germany.

*Antonio.Stocco@umontpellier.fr

\section{S1. Janus Particle Fabrication}

Figure S1(A) shows a large scale image of the Janus colloids fabricated by sputtering. Those particles are uniform with two distinct faces, i.e. silica and platinum (Figure S1(B)). The white regions displayed in Figure S1(B) represent the platinum layer. Platinum atomic percentage measured on one meridian of a Janus colloid is also shown in Figure S1(C). Negligible values are measured far from the white face of the colloid. The platinum content progressively increases from the equator to the pole, which agrees with the expected thickness profile of the platinum layer by sputtering. ${ }^{1}$ 

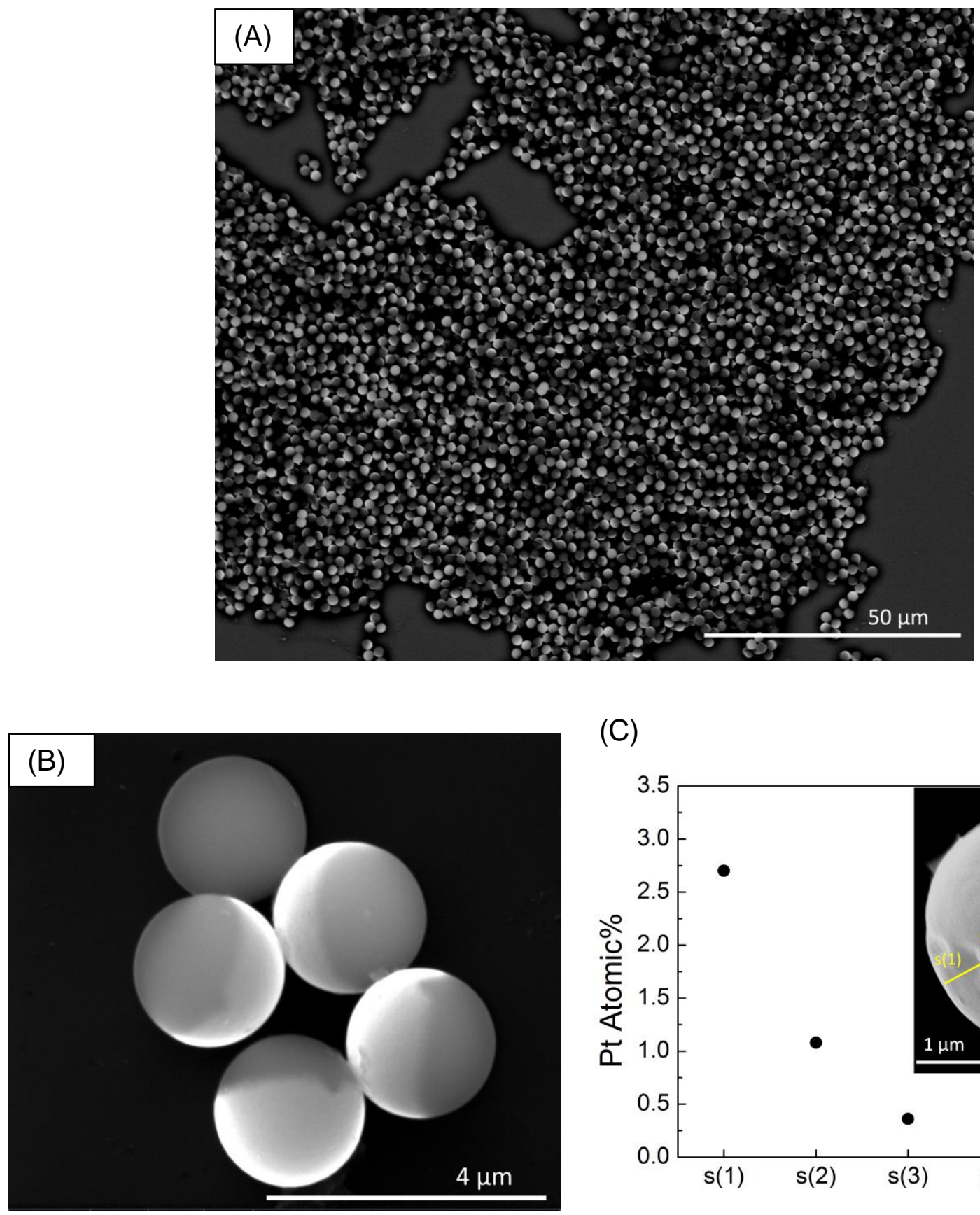

(C)

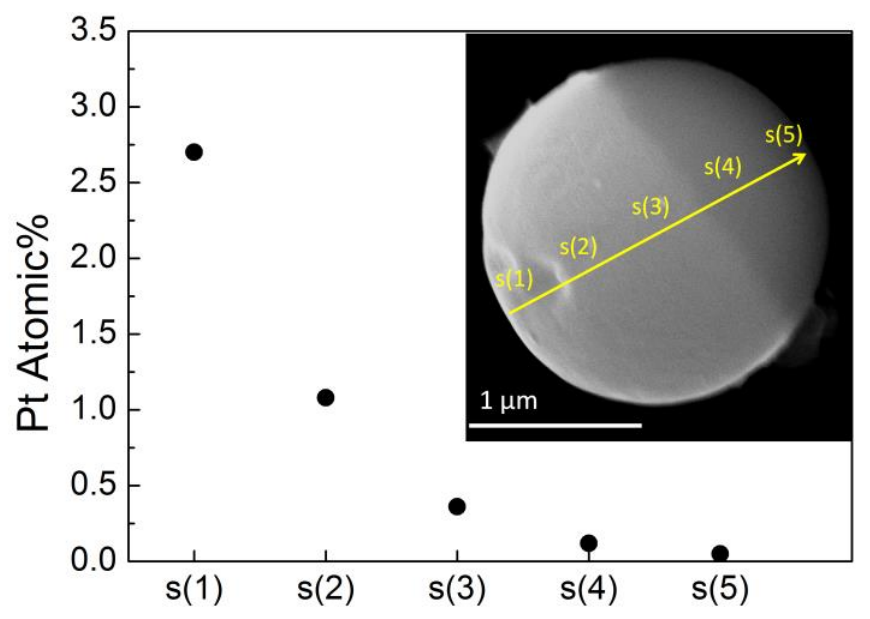

Figure S1. (A) SEM image of many Janus colloids fabricated by sputtering. (B) SEM image of five Janus colloids showing the white platinum layer (20 nm thick). (c) Platinum atomic percentage measured by SEM on five different spots s(i) on the Janus colloid. 


\section{S2. Contact angle by gel trapping}

Figure S2 shows SEM images of gel trapped colloids at the interface. Visible part of the colloids was previously immersed in gelled water.

Contact angle $\alpha$ can be calculated by $\cos \alpha=1-h / R$ (h is the height of visible bead and $R$ the radius). Averaging over more than 20 beads, we measured the contact angles of $\mathrm{SiO}_{2}$ bead: $70.9^{\circ} \pm 3.6^{\circ}$ and $\mathrm{Pt}-\mathrm{SiO}_{2}$ bead: $63.6^{\circ} \pm 2.2^{\circ}$.
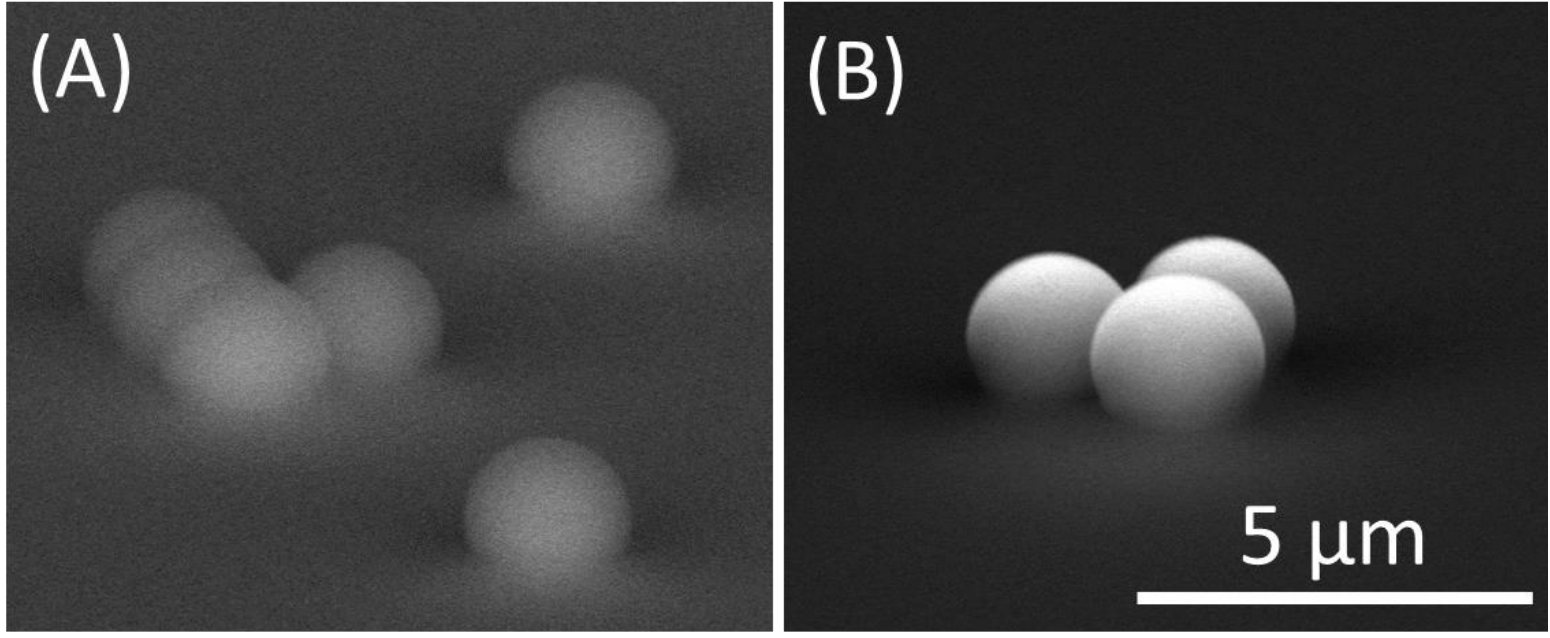

Figure S2. SEM images of (A) $\mathrm{SiO}_{2}$ beads, (B) Janus $\mathrm{Pt}_{-} \mathrm{SiO}_{2}$ beads trapped in polymerized NOA81 layers. The parts visible of beads were previously immersed in gelled water. 


\section{S3. Bulk measurements}

We measured trajectories and calculated the active velocity $V$ for active colloid immersed in the bulk at different $\mathrm{H}_{2} \mathrm{O}_{2}$ concentration (see also Figure $\mathrm{S} 4$ ). $V$ as a function of $\mathrm{H}_{2} \mathrm{O}_{2}$ concentration is shown in Figure S3. For comparison literature data are also shown.

Differences between our data and the ones reported previously may be due to (i) a different method used to evaluate the velocity, ${ }^{2}$ or (ii) given the weight of the colloid to a different distance to the solid wall of the glass container. In the latter case the hydrodynamic friction increases strongly when the distance between the particle and the wall decreases. ${ }^{3}$

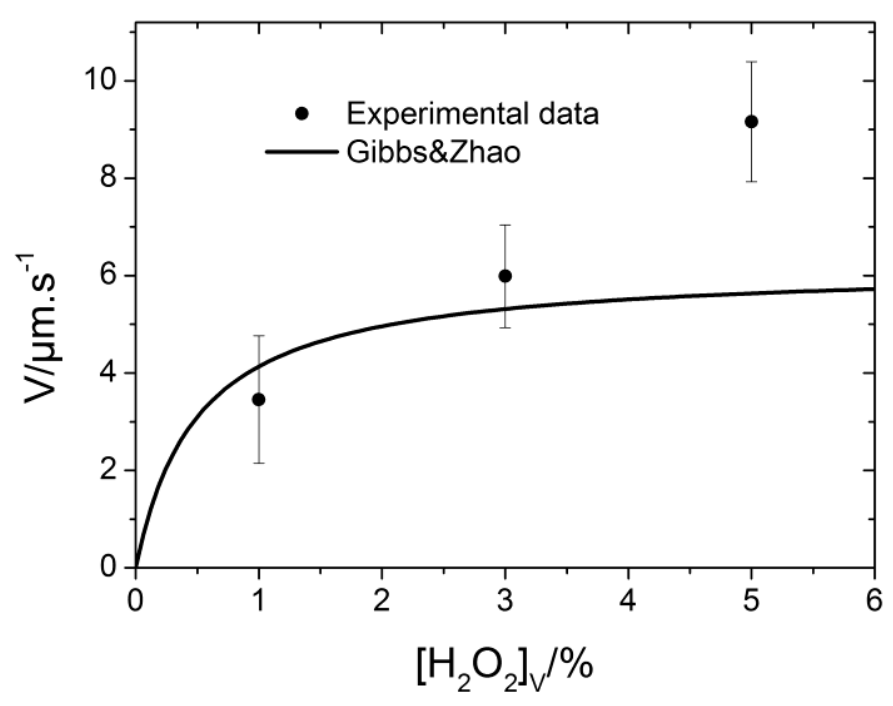

Figure S3. Active velocities from bulk experiments (filled circle). Solid curve is the fitting result of the data reported by Gibbs \& Zhao. ${ }^{2}$ 


\section{S4. Autocorrelation functions}

For Janus particles at the water surface, autocorrelation functions of the velocity vector as a function of delay time are shown in Figure S4.

For active colloids in bulk the autocorrelation function of the velocity can be written as: ${ }^{4}$

$$
\left\langle v(\Delta \mathrm{t}) \cdot v(0)>=4 D_{T, 0} \delta(\Delta \mathrm{t})+V^{2} \exp \left(-D_{R} \Delta \mathrm{t}\right)\right.
$$

where $4 D_{T, 0} \delta(\Delta \mathrm{t})$ corresponds to the passive Brownian motion contribution to the autocorrelation, $V$ is the active velocity and $D_{R}$ is the rotational diffusion. ${ }^{5,4}$

The quantity $\langle v(\Delta t) \cdot v(0)\rangle$ describes the probability of a colloid to keep the same velocity after a delay time $\Delta \mathrm{t}$. In the run and tumble model, this probability is lost when the colloid changes significantly its direction. If $\tau_{\mathrm{v}}$ is the characteristic time at which the directional change occurs, the probability becomes $\left(\tau_{\mathrm{v}}-\Delta \mathrm{t}\right) / \tau_{\mathrm{v}}$ for $\Delta \mathrm{t}<\tau_{\mathrm{v}}$ and the active term of the autocorrelation is: 5

$\langle v(\Delta \mathrm{t}) \cdot v(0)\rangle=V^{2}\left(\tau_{\mathrm{v}}-\Delta \mathrm{t}\right) / \tau_{\mathrm{v}}$

Data are fitted by equation 1 and 2 which lead to same values of the active velocity and rotational times within error bars.
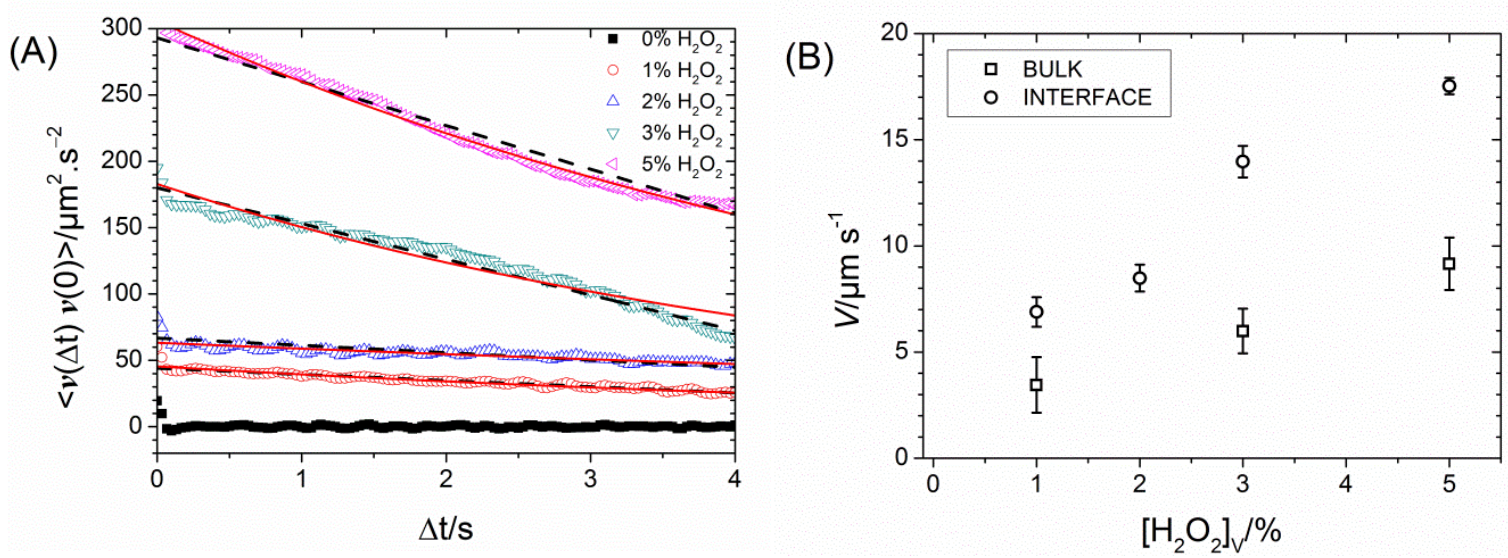

Figure S4. (A) Velocity autocorrelation functions for different $\mathrm{H}_{2} \mathrm{O}_{2}$ concentrations. Solid red lines are the fits of the data obtained with equation 1 . Dashed black lines are the fits of the data obtained with equation 2. (B) Active velocity $V$ at the interface (filled circle) and in the bulk (open squares) from the fit of the velocity autocorrelation function.

From the analysis of the velocity autocorrelation functions, the active velocity was evaluated for trajectories measured in a field of view of $477 \mu \mathrm{m} \times 358 \mu \mathrm{m}$, which agrees with the active 
velocity evaluated from the $M S D$ in a field of view $125 \mu \mathrm{m} \times 94 \mu \mathrm{m}$ (Figure 4). It increases with $\mathrm{H}_{2} \mathrm{O}_{2}$ concentration reaching $V \approx 18 \mu \mathrm{m} / \mathrm{s}$ at $\left[\mathrm{H}_{2} \mathrm{O}_{2}\right]_{\mathrm{V}}=5 \%$. For three different $\mathrm{H}_{2} \mathrm{O}_{2}$ concentrations, we have also investigated the motion in the bulk of the aqueous solution. When compared to the active velocity measured in the bulk, $V$ is approximately twice higher at the interface (Figure S4(B)).

\section{REFERENCES}

1 A. Brown and W. Poon, Soft Matter, 2014, 10, 4016-4027.

2 J. G. Gibbs and Y.-P. Zhao, Appl. Phys. Lett., 2009, 94, 163104.

3 A. J. Goldmans, R. G. Cox, H. Brenner and O. Neill, Chem. Eng. Sci., 1967, 22, 637.

4 S. Ebbens, R. A. L. Jones, A. J. Ryan, R. Golestanian and J. R. Howse, Phys. Rev. E Stat. Nonlinear, Soft Matter Phys., 2010, 82.

5 Z. Wang, H.-Y. Chen, Y.-J. Sheng and H.-K. Tsao, Soft Matter, 2014, 10, 3209-17. 